\title{
La escritura reflexiva para acceder a y potenciar la experiencia de lectura de un poema
}

\author{
Daniela Cavalli Munizaga \\ Universidad de Barcelona, Barcelona, España
}

(Artículo recibido el 27 de junio de 2011; versión final recibida el 24 de agosto de 2011)

En este artículo se da cuenta de los resultados de una investigación desarrollada como trabajo final de máster que ha servido de base para un proyecto de tesis doctoral. La investigación, de carácter cualitativo, indagaba sobre las posibilidades que la escritura reflexiva e intermediaria ofrecía para acceder a y estimular la experiencia de lectura de un poema con alumnos de secundaria. A partir del análisis de seis textos escritos por tres estudiantes, se observó que este tipo de escritura permitía obtener conocimiento sobre la vivencia personal de lectura y que podía contribuir al desarrollo de la competencia literaria específica al género poético. También, se dio cuenta de problemáticas que trascendían el terreno de la poesía y el de la escritura reflexiva, pero que podían afectar los procesos de enseñanza y apendizaje involucrados.

\section{Introducción}

El presente trabajo de investigación, que ha posibiltado el planteamiento de un proyecto de tesis doctoral, ha nacido de nuestro interés por trabajar para que alumnos y alumnas tengan la oportunidad de 'descubrir' la poesía y de beneficiarse de todo el potencial de desarrollo cognitivo, afectivo y cultural que ésta les ofrece. Conseguir esto puede ser una tarea muy difícil en el interior de un aula. Por lo mismo, consideramos relevante el desarrollo de estudios que indaguen en y den cuenta del valor de la poesía en el contexto de la educación secundaria actual y que éstos se realicen desde la Didáctica de la lengua y la literatura.

Con el objeto de conocer más sobre el desarrollo de la competencia literaria para la poesía, este estudio ${ }^{1}$ indagó en las posibilidades que la escritura reflexiva ofrecía para, en parte, acceder a y estimular la experiencia de lectura de poesía de estudiantes de secundaria.

Se da cuenta de los resultados de una investigación llevada a cabo en una escuela concertada de Barcelona, en un aula de "Llengua catalana i literatura" de tercero de la ESO. La 
propuesta didáctica realizada a petición nuestra, por la profesora de dicha clase, consistió en un trabajo con poesía que facilitó que los alumnos escribieran textos reflexivos.

Además de delimitar el área de interés de esta investigación, la revisión bibliográfica llevada a cabo nos permitió ampliar nuestro entendimiento en tres áreas: la didáctica de la literatura/poesía, el rol de la motivación y del esfuerzo en el desarrollo de las actividades en el aula y la educación de la reflexividad.

En lo que concierne específicamente a la enseñanza-aprendizaje de la poesía, los trabajos de Bordons y Díaz-Plaja (2005), Bordons (2009) y Ribeiro (2009, 2010) nos ayudaron a ver con más claridad algunos desafíos que se enfrentan al trabajar la poesía en el aula (especialmente en relación a la selección de textos y de actividades). Por otro lado, también, pudimos reafirmar nuestra creencia en el valor que la poesía tiene en la formación de la persona. En relación a esto, creemos fundamental lo postulado por Ribeiro (2010) cuando habla del valor intrínseco de la poesía y de éste desprende su valor instrumental, no a la inversa.

Respecto al rol de la motivación y del esfuerzo en el desarrollo de las actividades en el aula, nos interesó el trabajo de Witte, Janssen y Rijlaarsdam (2006) por la amplitud de su visión. En su estudio sobre formación de la competencia literaria, señalan que la actitud del lector competente está en parte definida por su voluntad y su deseo de invertir en la lectura, proponiendo, así, la motivación como categoría de análisis de la competencia literaria. En cierta coincidencia con lo postulado por estos autores, pero desde la psicología educacional, la investigación de Blackwell, Dweck y Trzesniewski (2007) sobre las concepciones de inteligencia que subyacen a la valoración que los alumnos hacen del esfuerzo ofreció un contexto más amplio desde el cual mirar la motivación. Estas autoras hablan de la importancia de las actitudes hacia el trabajo escolar (no sólo en el aula de lengua y literatura), por la repercusión que éstas tienen en el nivel de esfuerzo con el que los estudiantes realizan sus actividades.

La educación de la reflexividad la miramos en concordancia con los aportes de las teorías constructivistas que hablan de la importancia -para el aprendizaje- de dar espacio (darles la palabra) a los alumnos a fin de que sean ellos quienes resuelvan, gestionen, elaboren, piensen y que no sea el profesor quien, en su afán 'instruccional', monopolice no sólo la palabra, sino también la reflexión (Lefranc, 2004; Nussbaum, 1996). A la luz de estos aportes, entendimos que 
el diálogo en el aula ha de reconocer al otro como un interlocutor válido y que el carácter asimétrico del diálogo, en la dimensión profesor-alumno, no le restaría valor a este último.

Concretamente, vimos una oportunidad para la educación de la reflexividad, aplicable al desarrollo de la competencia literaria para la poesía, en la propuesta Chabanne y Bucheton (2002) y Chabanne (2005). Estos autores y su equipo han desarrollado una interesante investigación en relación a lo que denominan la escritura reflexiva y el texto intermediario. En su propuesta se refieren principalmente a la reflexividad lingüística:

[...] l'entrelacement de différents plans: cognitifs, socio-affectifs, linguistiques, etc. entrelacement dynamique et créatif sur le plan verbal par laquel le sujet sémiotise son expérience, la culture rencontrée, les discours entendus. Ni séquentiel ni modulaire, ce processus langagier réflexif reste singulier, divers et imprévisible. On constate qu'il instrumente le sujet dans sa capacité à penser. (Chabanne y Bucheton, 2002: 42)

Esta escritura reflexiva se desarrolla en el texto intermediario, el instrumento de mediación: “[...] intermédiaire entre deux états d’un écrit à mettre en forme, entre deux états de pensée, entre les membres d'un groupe de travail, entre des écrits et des oraux, etc. [...]" (Chabanne y Bucheton, 2002: 20)

De lo planteado por ellos nos interesó la valoración de los procesos de pensamiento y de construcción identitaria que pueden desarrollarse a partir de las prácticas lingüísticas escolares. Al darle espacio y tiempo al sujeto para su expresión y formación se facilita su implicación en el trabajo escolar y se reconoce su poder y potencial como aprendiente. Además, nos pareció importante lo que muestran sobre el rol formativo (intelectual y afectivo) que la escritura 'en espiral' puede tener. Estos autores constituyeron un referente fundamental no sólo a nivel teórico, sino que también metodológico. En nuestro estudio, se buscó que los alumnos escribieran textos de carácter reflexivo e intermediario que, principalmente, entendimos en los términos propuestos por ellos.

La escritura reflexiva, sin embargo, también la entendimos a partir del trabajo con los relatos de vida lingüística que hace el grupo PLURAL de la Universitat de Barcelona. En la consideración, desde la educación, que se hace de la propuesta de los etnosociólogos (Bertaux, 1997) se entiende que el relato de vida favorece la toma de conciencia sobre el propio repertorio 
lingüístico. Aquí, lo dicho se aplicó en la utilización del escrito que reflexiona sobre la lectura de un poema. Si bien nuestros textos no eran relatos de vida, se podría decir que tenían algunas de sus características: relevancia identitaria por contar una experiencia vivida (la de lectura), contar a petición de otro y el potencial de facilitar la toma de conciencia como 'lector de poesía’.

Por el valor que le otorgamos y por no haber hallado estudios que lo consideraran, nos propusimos el objetivo de observar los efectos que esta escritura reflexiva puede tener en el desarrollo de la competencia liteararia para la poesía. Las posibilidades visualizadas en este tipo de escritura se unieron al hecho de que no encontramos información relativa a la vivencia personal e interna de la lectura de poesía en alumnos de secundaria. Fue así que estimamos pertinente plantearnos la obtención de conocimiento en esta área como otro de los objetivos de investigación.

Las preguntas que guiaron nuestras indagaciones fueron: ¿qué de la experiencia de lectura de poesía se revela en la escritura reflexiva? y ¿cómo potencia la escritura reflexiva la experiencia de lectura de poesía, contribuyendo, así, al desarrollo de la competencia literaria para la poesía? ¿se presentan dificultades?

La opción metodológica cualitativa de este estudio se ha fundado en la creencia de que, para observar en profundidad un fenómeno y para entender el microcosmos en el que interactúan los participantes, es necesario acercarse al escenario en el que naturalmente ocurre el hecho social.

Para la elicitación de datos y su interpretación, buscamos los métodos que nos brindaran un mayor potencial de acceso a la interioridad de los involucrados, que fueran aplicables al escenario escogido y, sobretodo, que fueran adecuados para observar el fenómeno de la reflexión en torno a la lectura poética. Éstos fueron: la observación participante -participante en el sentido de implicada (Cambra, 2003)- y la escritura de textos reflexivos por parte de los alumnos, entendida de acuerdo a los referentes ya explicados. La interpretación la construimos haciendo un análisis del discurso de los textos escritos por los estudiantes.

Para poder tratar los datos en profundidad y por creer que la mirada microscópica nos permite acceder a lo general a través de lo particular (Bertaux, 1997) el corpus que efectivamente se analizó fueron seis textos escritos por sólo tres alumnos de la clase. Cada alumno escribió dos 
textos, el primero (txt1), durante la clase, después de haber leído por primera vez el poema "La poma escollida” (Carner, 1984 [1906]). El segundo (txt2) fue escrito después de una discusión en grupo en la que compartieron sus escritos y hablaron sobre el poema. Dicha interacción ocurrió en la clase que siguió a la de la lectura del poema y la escritura del txt1. La escritura del txt2 se llevó a efecto como tarea para la casa. Las instrucciones dadas por la profesora para la escritura del txt1 fueron: que leyeran el poema en forma individual y que escribieran lo que cada uno sacaba de él, lo que le sugería. Luego de observar brevemente a los alumnos, la profesora leyó el poema en voz alta y dijo que dejaran la métrica de lado para abocarse a lo que éste decía. Los alumnos tuvieron aproximadamente veinte minutos para escribir. Para la escritura del txt2, la profesora pidió que apuntaran las palabras clave de la discusión y que, en casa, re-escribieran el texto considerando lo puesto en común y lo que todo esto te ha hecho pensar. Los alumnos, aquí llamados Ariel, Alex y Lluïsa, integraban uno de cuatro grupos de conversación sobre el poema.

\section{Discusión general de los resultados}

El análisis de los datos fue realizado con una mirada amable o "regard positif” (Chabanne y Bucheton 2002:44), es decir, con la intención de valorar el proceso de reflexión en cualquier estadio en que éste se encontrara y de buscar el sentido que el alumno daba a sus palabras. Evitamos leer los textos desde la perspectiva 'correctiva' con que los profesores muchas veces enfrentamos el trabajo de los alumnos. No obstante, creímos importante señalar los avances del proceso de reflexión, incluyendo las dificultades de comprensión lectora.

El análisis interpretativo de los textos de los alumnos en su conjunto nos permitió organizar la información en tres grandes ámbitos interrelacionados: poesía, escritura reflexiva y textos intermediarios y representaciones del trabajo.

\section{Poesía}

A continuación referiremos lo que la tarea de escritura reflexiva reveló sobre la experiencia de lectura de poesía desde la perspectiva de la enseñanza-aprendizaje de ésta.

Un primer aspecto relevante fue que, en el txt1, los alumnos hicieron comentarios sobre los recursos estilísticos del poema sin explicar ni establecer relaciones con su contenido y, a 
veces, incluso con errores. Esto nos llevó a suponer que la mayoría de los estudiantes comprende poco la articulación entre ‘forma’ y ‘contenido’ y que entienden que la mención a los recursos estilísticos es algo así como una primera etapa obligatoria del análisis de un poema. Sin embargo, es posible que algunos alumnos estuvieran comenzando a apreciar el sentido que tiene conocer sobre recursos estilísticos (lo vimos en un caso). El hablar del poema con un lenguaje que usa palabras como ‘metáfora’ y ‘comparación’², para designar fenómenos que no podrían nombrarse con la misma exactitud de no existir estas palabras, muestra una apropiación real de los conceptos.

En segundo lugar, considerando la forma en que, hemos visto, más habitualmente se enseña-aprende poesía en secundaria, nos preguntamos si esa forma de tener en cuenta los recursos de estilo ayudaba o, más bien, dificultaba el acercamiento de los alumnos al poema. Esta problemática, que no pudimos responder, cobra mayor complejidad si se piensa que estos recursos 'en uso' pueden facilitar el acceso al poema. La lectura, que actualiza los sonidos de un poema y que, por ende, da vida a sus recursos fónicos, ayuda a su comprensión (Ribeiro, 2009, 2010). El poema se devela con distintos matices en la lectura silenciosa, en la lectura en voz alta, escuchando a otro leer o recitar. Además, vale la pena considerar que la cognición vía auditiva es diferente a la visual y que la comprensión del significado de una palabra es diferente a la de su musicalidad.

Otro tópico de interés aportado por los datos analizados tiene que ver con la cercanía respecto de la temática del poema. El motivo central del poema seleccionado hizo difícil, a nuestro entender, la identificación personal inmediata de los alumnos (el poema habla del amor entre dos ancianos). Sin embargo, éstos buscaron una forma de acercarse y creemos que esta posibilidad estuvo dada, en gran medida, por las instancias de reflexión. La invitación a que el poema les sugiriera algo a nivel personal ayudó a que pensaran en los propios abuelos o en el amor (las formas diferentes en que lo viven viejos y jóvenes; el 'amor eterno').

Hem parlat sobre l'amor dels yayos, que es molt diferent al dels joves. Perque per a ells l'amor es estar la resta de la seva vida amb una persona, i per als joves l'amor es estar llunts sense pensar en estar la resta de la vida amb l'altre. (Ariel, txt2) 
Un amor que dura anys, fins a la mort. ¿Hi ha un amor que arribi intacte fins a l’hora de la mort? Realment, no ho sé. I espero que si per que aixó té que ser molt bonic. Tant com per fer fora tots els pors i neguits fora. (Lluïsa, txt2)

La discusión a partir del poema llevó a los alumnos a hablar de las situaciones familiares de cada uno (abuelos) y no a ocuparse exclusivamente de lo que durante la observación (de sus otras clases de poesía) vimos que comentaban (recursos estilísticos, tema central y posible vinculación del poema/autor a un movimiento artístico-social). La actividad realizada abrió un espacio para compartir a nivel social la experiencia personal: "Hem parlat si els nostres avis els hi pasava el que pasava al poema, però ninguns avis dels alumnes del meu grup no li pasa això.” (Alex, txt2).

Por un lado, la lejanía en relación a la temática del poema podía ser un obstáculo, pero por otro, un beneficio, ya que permitía tomar distancia de uno mismo para pensar en otros aspectos de la realidad. El valor universal de muchas de las temáticas encontradas en la literatura y la relevancia cultural de otras permiten que 'el lector' enfrente problemáticas que ayuden a su formación moral. Lo vimos en los textos de los tres alumnos: Ariel se cuestiona las actitudes ante los abuelos; Alex finaliza su reflexión extrayendo una suerte de moraleja y Lluïsa cuestiona los valores sociales que se le otorgan al amor.

La poesía es un repertorio de vivencias humanas que nos sirven como pequeños espejos donde ir reflejando las nuestras. La naturaleza metafórica del lenguaje no poético (Lakoff, 1980) también abre espacios para significar e interpretar. Por lo tanto, la poesía, que usa el lenguaje común de por sí metafórico, tiene un enorme poder de significación ${ }^{3}$.

\section{Escritura reflexiva y textos intermediarios}

En este segundo apartado, nos referimos a los efectos e implicaciones de la escritura reflexiva en el desarrollo de la competencia literaria para la poesía.

Teniendo en cuenta los aportes de Chabanne y Bucheton (2002) y Chabanne (2005), se podría decir que tanto los primeros como los segundos textos escritos por los alumnos tuvieron un estatus intermediario (los primeros más que los segundos). Algunas de las características de estos escritos encontradas en nuestros datos fueron: el comenzar la tarea de escritura sin 
introducción; los finales reflexivos y abiertos; la poca atención a la ortografía y a las reglas gramaticales; la estructura textual poco cohesionada y, muchas veces, de apariencia inconexa (exceso de "també” y otros nexos que adicionan sin jerarquizar); el poco desarrollo de las ideas y su escaso soporte textual (citas, ejemplos), los cambios en el tono de la enunciación (más o menos distante); el dar por descontada la colaboración del lector en la compleción del sentido del texto.

También a la luz de los aportes hechos por los autores citados, diríamos que el carácter reflexivo de la escritura se observó en, primero, el uso de indicadores textuales que denotaban un equilibrio inestable entre escribir para sí mismo, para la profesora y para la escuela: "la frontière du privè et du scolaire” (Chabanne, 2005:62).

Segundo, el escribir para entender el poema. En el txt1 se ve que esta primera aproximación los llevó a hacer el esfuerzo de comprender por sí mismos. Sin embargo, en el txt2 se ve que el escribir para integrar otros puntos de vista y para reformular los propios los lleva a profundizar la comprensión. El segundo texto, que en los tres casos evidenció un estadio de comprensión más avanzado, de alguna forma menciona y recoge la instancia de reflexión grupal.

Lo dicho en este último párrafo puede apreciarse en los siguientes ejemplos:

\section{Ejemplo I (Ariel)}

Ells cada vegada es senten pitllors fisicament. Al ser tan vells no s'enrecorden de coses passades. La avia es sent malament perque els seus néts la troben gelada, vella, corbada i seca. (txt1)

També hem parlat sobre la tristesa que tenen els yayos perque es senten inútils. (txt2)

\section{Ejemplo II (Alex)}

Aquest poema va d'una noia vella, i un noi vell. Que la dona es sent corbada i está parlant i el noi el consola. (txt1)

També em parlat sobre de que parla el poema, em decidit que parlava d'uns avis, que s’estiman molt i tambe de tristeza perque es sent corbada. (txt2)

Ejemplo III (Lluïsa)

Sempre són junts, no volen separar-se pot ser per culpa del por o per que volen está jonts mentre envellesen. També em transmet impotencia per no poder fer les coses com voldrien. (txt1) 
A la reflexió que he arribat avui es que, realment ningú de nosaltres a sapigut arribar al fons d’aquest poema. Era l'amor, si, pero aquest amor amagaba també inquietud. (txt2)

Tercero, y como se ve en los ejemplos, los datos también sugieren problemas de comprensión lectora. Las explicaciones del poema que usaban su lenguaje figurado como si no lo fuese, evidencian una dificultad para reformular o parafrasear. El siguiente fragmento del poema ayuda a verlo mejor.

-Oh petita Alidé, com és que plores tant?

-Oh Lamon, perquè em sé tan vella i tan corbada

i sempre sec, i envejo les nores treballant, i quan els néts em vénen em troben tan gelada.

\section{Representaciones del trabajo}

En nuestro análisis de los datos encontramos una tercera dimensión a partir de la cual entender lo observado, la de las representaciones que tenían los alumnos del trabajo a realizar. Ésta se manifiestó, en la imagen y en la experiencia previa que ellos tenían del quehacer escolar, en los esquemas mentales con que entendían la poesía, en general, y a través de la interpretación que hicieron de las instrucciones recibidas.

En primer lugar, vimos que los datos sugerían que los alumnos enfrentaron la secuencia de trabajo: lectura del poema- escritura reflexiva- interacción grupal- escritura reflexiva, guiándose, en gran medida, por sus experiencias previas en relación al trabajo escolar (en esta clase) y a las expectativas que esto generó (Tannen, 1993; Woods, 1996). Las instrucciones dadas por la profesora, quizás pidieron hacer algo que no sabían bien cómo hacer. Esto explicaría las oscilaciones entre hablar del poema y hablar, explícitamente, de la propia lectura de él, entre el discurso argumentativo y el expresivo, entre incluir referencias a los recursos de estilo y el referirse a los propios sentimientos.

El espacio dado al análisis de los elementos estilísticos del poema parecía estar modelado por la forma en que habían trabajado la poesía en la clase. La brevedad y, en gran parte de los casos, la simplicidad de las reflexiones pueden deberse a que en general en su clase no acostumbraban a destinar un tiempo prolongado a reflexionar sobre los contenidos de los poemas 
leídos y a que el trabajo propiamente reflexivo tendía a ser realizado por la profesora. Los alumnos tenían un rol enunciativo bajo a la hora de resolver los desafíos cognitivos presentados (Lefranc, 2004; Nussbaum, 1996). Cabe, sin embargo, tenerse en cuenta que nos basamos en la observación de clases realizada durante sólo un mes.

Por otro lado, es posible que los estudiantes no quisieran hacer un trabajo que requiriera de más esfuerzo, aunque supieran cómo hacerlo. Esto se sustenta en la observación de una serie de actitudes y comportamientos en relación a sus deberes como estudiantes. En general, no trabajaban mucho en sus tareas para la casa y, en clases, a menudo, se distraían y buscaban formas de evadir el quehacer. Traspasando los márgenes de este estudio y a la luz de lo postulado por Blackwell, et al. (2007) vale la pena preguntarse si entre estos alumnos predominaría -a nivel de cultura escolar- una concepción fija de inteligencia, algo que se tiene o no y que, en consecuencia, no se alcanza al esforzarse más.

Las instrucciones recibidas y la interacción grupal previa situaron el discurso elaborado en los txt2 en un contexto de producción específico. Al pedirles, la profesora, que recogieran las palabras más importantes de la discusión, para que en casa la recordaran, ¿les dio una herramienta que los ayudó o que les restó autonomía? Estas palabras clave, en distinta medida, permitieron articular el segundo texto a nivel estructural y temático en los tres casos.

Los esquemas de representación mental de los alumnos (de la clase de catalán, de la poesía, etc.) permiten que éstos puedan comprender e interactuar, sin embargo, a la vez podrían estar limitando la ampliación o la ruptura y reestructuración de los mismos (Bruner, 1990; Stockwell, 2002). La observación de estos fenómenos socio-cognitivos es uno de los puntos que nos interesa abordar en la investigación doctoral que estamos desarrollando.

\section{Conclusiones}

Los resultados obtenidos, a la luz de la teoría en la que nos hemos apoyado, de nuestro propio interés y de lo aprendido durante la realización de este estudio nos permitieron llegar a algunas conclusiones que, más que cerrar, abrieron otra etapa de reflexión.

Se cumplieron, con limitaciones, los dos objetivos fijados. En primer lugar, los textos escritos por los alumnos nos permitieron obtener cierto conocimiento sobre la vivencia personal e 
interna de la lectura de poesía de alumnos en un contexto de educación secundaria. En segundo lugar, pudimos observar efectos posibles de la escritura reflexiva en el desarrollo de la competencia literaria para la poesía.

En respuesta a la pregunta de investigación: ¿qué de la experiencia de lectura de poesía se revela en la escritura reflexiva? Observamos, primero, que las representaciones que los alumnos tienen con respecto al trabajo escolar y a la poesía son influyentes y quizás predominantes a la hora de darle sentido por escrito a un poema. Incluso la interpretación de las instrucciones para la escritura parece estar influida por estas representaciones mentales.

Segundo, vimos que la apreciación de los aspectos formales del poema está modelada por estas representaciones preexistentes y no parece haber conciencia del valor que éstos tienen en el sentido que puede alcanzar el poema a nivel cognitivo, afectivo y social.

Por último, apreciamos la importancia del tema para generar reflexión. ${ }^{4}$ La reflexión es mayor o menor de acuerdo a la posibilidad de acercarse a la temática del poema. Vimos que los alumnos buscaron formas de conectarse con el poema, pese a que su contenido parecía poco relevante para ellos. Como se ha dicho, esto puede responder a la invitación a vincularse. Los motivos de un poema no sólo permiten la identificación, sino que también pueden hacer visible 'al otro'. No obstante, el potencial reflexivo que brinda una temática próxima puede no ser suficiente. La mediación docente ayuda.

En relación a las preguntas: ¿cómo potencia la escritura reflexiva la experiencia de lectura de poesía, contribuyendo, así, al desarrollo de la competencia literaria para la poesía? ¿se presentan dificultades? Entendemos que la escritura de textos intermediarios genera reflexión tanto al hablar del poema como al hablar de la propia lectura de él. Por lo mismo, creemos que la escritura reflexiva en textos intermediarios sí potencia la experiencia de lectura poética y, en consecuencia, el desarrollo de la competencia literaria. La comprensión se profundiza al haber nuevas oportunidades para reflexionar. Para que éstas sean tales, parece ser relevante la mediación (instrucciones; actividades). La escritura es una herramienta, entre otras, que puede activar el potencial reflexivo de un poema. La interacción grupal también lo es.

Se vio que la escritura entendida como intermediaria creó un espacio de libertad expresiva y entregó un rol enunciativo más alto los alumnos. Sin embargo, no quedó claro cuánto escribían 
para explicarle a la profesora o para ellos mismos entender. El rol enunciativo más activo que asumieron los tres alumnos en el segundo texto fue, para nosotros, un aporte del proceso reflexivo generado en esta actividad. Sin embargo, el carácter intermediario tanto de los primeros textos como de los segundos mostró, por un lado, la necesidad de etapas de escritura o reflexión posteriores para el alumno y, por el otro, la necesidad del seguimiento docente. En este caso, la actividad no tuvo la continuidad y el cierre que requería.

Con nuestros datos no se pudo explicar si la mayor comprensión del poema que se ve en los segundos textos de los alumnos fue fruto de la evolución natural de su proceso de lectura y pensamiento, si fue generada por la interacción entre pares o si respondió a un mejor planteamiento de las instrucciones por parte de la profesora y qué rol tuvo el escribir en esta evolución. Tampoco fue posible diferenciar las voces de los alumnos en los segundos textos. Éstas son limitaciones de la escritura reflexiva como instrumento para la recolección de datos, no como herramienta de desarrollo de la competencia literaria.

Quedaron abiertas algunas interrogantes. El trabajo propuesto a los alumnos los invitaba a revelar parte de su mundo privado, ¿ंcruzaron "la frontière du privè et du scolaire" (Chabanne 2005:62)? Además, este trabajo requería de esfuerzo, ¿cuánto se esforzaron? ¿contamos con la voluntad y el deseo de invertir en la lectura del que Witte et al. (2006) hablan? ¿Ampliaron/reformularon los alumnos sus representaciones mentales del trabajo realizado?

Las instrucciones de la profesora no explicaron la finalidad de la actividad. Fue responsabilidad nuestra que no ocurriera, ya que por temor a imponer un trabajo didáctico y en nuestro intento de hacer participar a la profesora, no fuimos lo suficientemente claros en relación al objetivo de la actividad. A raíz de esto, surgió la siguiente pregunta metodológica: ¿¿cuáles son los límites de la participación en un escenario?

La investigación que aquí se ha presentado intenta hacer un pequeño aporte a quienes se interesan por la enseñanza y el aprendizaje de la poesía. No obstante, llevando nuestra búsqueda a una nueva etapa, esperamos poder ampliar y profundizar el conocimiento de la experiencia de lectura de poesía e ir respondiendo algunas de las preguntas que aquí han quedado abiertas. 


\section{Referencias bibliográficas}

Bertaux, D. (1997). Los relatos de vida. Paris: Nathan. 69

Blackwell, L., Trzesniewski, K, \& Dweck, C. (2007). Implicit theories of intelligence predict achievement across an adolescent transition: a longitudinal study and an intervention. Child Development, 78 (1): 246-263.

Bordons, G. (Ed.) (2009). Poesia i educació. D’internet al aula. Barcelona: Graó.

Bordons, G., y Díaz-Plaja, A. (Eds.) (2005). Enseñar literatura en secundaria. La formación de lectores críticos, motivados y cultos. Barcelona: Graó.

Bruner, J. (1990). Acts of meaning. Cambridge, MA: Harvard University Press.

Cambra, M. (2003). Une approche ethnographique de la classe de langue. Paris: Didier.

Carner, J. (1984, $2^{\text {nd }}$ ed.). Els fruits saborosos. Barcelona: Edicions 62 (col. L'alzina 1).

Chabanne, J.-C. et Bucheton, D. (2002). Parler et écrire pour penser, aprendre et se construire. L'ecrit et l'oral réflexifs. Paris: Presses Universitaries de France.

Chabanne, J.-C. (2005). Écritures réflexives, la construction de la pensée et des connaissances chez les élèves d’école primaire. En: Molinié, M. et Bishop, M.-F. (ed.) Autobiographie et réflexivité (pp. 53-68). Cergy-Pontoise: Encrage/Les Belles Lettres.

Colomer, T. (1996). La didáctica de la literatura: temas y líneas de investigación e innovación. En: Lomas, C. (Ed.) La educación lingüística y literaria en la enseñanza secundaria, (pp.123-142). Barcelona: ICE, Universitat de Barcelona y Horsori.

Lakoff, G. y Johnson, M. (1980). Metaphors we live by. Chicago: University of Chicago Press.

Lefranc, Y. (2004). FLE, FL “M”, FLS: Les apprenants, leur faculté de langage et la classe de langue. ELA: études de linguistique appliquee: revue de didactologie des languescultures et de lexiculturologie, 133: 79-96.

Nussbaum, L. (1996). La didáctica de la lengua: temas y líneas de investigación e innovación. En: Lomas, C. (Ed.) La educación lingüística y literaria en la enseñanza secundaria, (pp. 109-122). Barcelona: ICE, Universitat de Barcelona y Horsori.

Ribeiro, J.M., (2009). El valor pedagògic de la poesia. En: Bordons, G. (ed.) Poesia $i$ educació. D’internet al aula (pp. 15-25). Barcelona: Graó.

Ribeiro, J.M., (2010). O papel da poesia na formação da pessoa. En: Bordons, G. (ed.) Poesia contemporània, tecnologies i educació (pp. 113-120). Barcelona: Universitat de Barcelona.

Stockwell, P. (2002). Cognitive poetics. London: Routledge.

Tannen, D. (1993). What's in a Frame? Surface evidence for underlying expectations. En: Tannen, D. (Ed.) Framing in discourse (pp.14-56). New York: Oxford University Press.

Witte, T., Janssen, T., \& Rijlaarsdam, G. (2006). Two empirical studies to describe the variation in the literature curriculum and the development of literary competence in upper secondary education in the Netherlands. Presentado en The International Colloquium Mother Tongue Education in a Multicultural World: Case Studies and Networking for Change, 22-25 June 2006, Sinaia, Rumania.

Woods, D. (1996). Teacher cognition in language teaching. New York: Cambridge University Press. 
1 Este estudio fue presentado (y publicado digitalmente), en una versión diferente, en el Congreso internacional reinventar la profesión docente de la Universidad de Málaga en Noviembre de 2010.

2 Parece ser que los conceptos de ‘metáfora’ y ‘comparación’ se internalizan más fácilmente por su presencia en el lenguaje cotidiano.

3 A lo largo de este apartado, nuestras palabras y reflexiones se sustentaron en Bordons (2009), Ribeiro (2009, 2010) y, de alguna forma, también en Colomer (1996).

4 Chabanne y Bucheton (2002) hablan de la implicación simbólica.

\section{Referencias de la autora:}

Daniela Cavalli Munizaga es estudiante del doctorado en didáctica de la lengua y la literatura de la Universidad de Barcelona. Su línea de investigación es la educación literaria, específicamente, se interesa por el pensamiento sobre poesía y la experiencia de su lectura en alumnos de secundaria. Trabaja con una metodología cualitativa, que constituye otra área en la que le interesa desarrollar reflexión. Es profesora de castellano y ha trabajado en escuelas secundarias en Chile y en los Estados Unidos. También se ha desempeñado como profesora de inglés. Actualmente, participa en dos grupos de investigación: POCIÓ (Poesia i Educació) y PLURAL (Plurilingüismes escolars i aprenentage de llengües), ambos de la Universidad de Barcelona.

Email: danielacavalli@hotmail.com 